\title{
Proposed emerged and enhanced routing protocols for wireless networks
}

\author{
Dheyaa Jasim Kadhim ${ }^{1}$, Ali Abdulwahhab Mohammed ${ }^{2}$ \\ ${ }^{1}$ Department of Electrical Engineering, University of Baghdad, Baghdad, Iraq \\ ${ }^{2}$ Department of Remote Sensing, Al-Karkh University of Science, Baghdad, Iraq
}

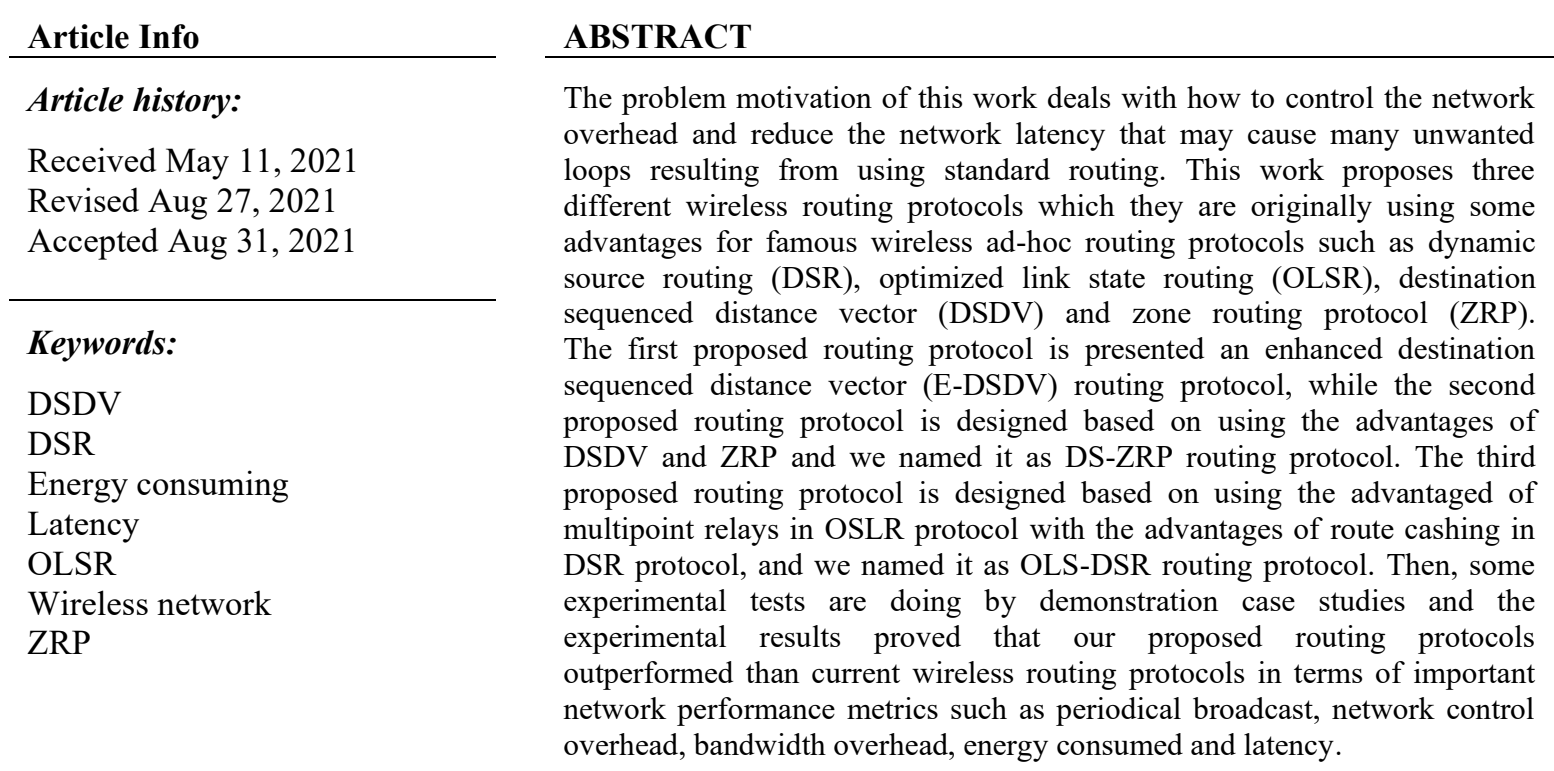

This is an open access article under the CC BY-SA license.

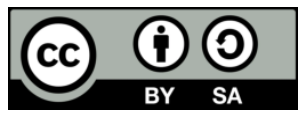

Corresponding Author:

Dheyaa Jasim Kadhim

Department of Electrical Engineering

University of Baghdad

Karrada, Al-Jadriya, Baghdad, Iraq

Email: dheyaa@coeng.uobaghdad.edu.iq

\section{INTRODUCTION}

Wireless networks aimed toward meeting specific service necessities whereas handling specific transmission impairments and optimizing the use of the system resources to confirm cost-effectiveness and satisfaction for the user [1]. Taking into consideration the important features of wireless nodes such as low power, high throughput and less latency time, therefore, it will be proposed to design the routing protocols for the purpose of forwarding the routing request to the intended destination node so as having better whole network performance and thus improving the performance of the network applications [2]. The most important challenges in designing routing protocol of a wireless network is reducing the energy consumption and increasing network lifetime [3], [4]. Some routing protocols have to achieve some quality of service (QoS) metrics e.g. high throughput, less node delay, secure communication and large covering [5], [6]. Routing protocols in wireless networks should enhance the system performance in term of energy-efficiency, network bandwidth, network overhead control and latency without deploying many unwanted or wasted loops in the network with simple routing flooding schemes deployment so it can reduce number of hops and 
the cost significantly as well offering high throughput and minimizing latency issue [7]-[9]. As known latency is the key obstacle for performance improvement of wireless systems, therefore best proposal routing protocol is one candidate to tackle/circumvent this shortcoming. Some related works such as [10]-[14] with aggressive adding more enhancements to standard wireless routing protocols as well network performance improvement, the previous standards of conventional routing protocols (destination sequenced distance vector (DSDV), dynamic source routing (DSR), optimized link state routing (OLSR), and zone routing protocol (ZRP)) did not take care more attention about energy-efficiency issue and the capacity of those conventional routing protocols are limited principally due to scarcity of bandwidth. Therefore, by developing those conventional routing protocols, the traditional wireless systems can reduce the network overhead that will reduce significantly the node and network latency for specified bandwidth [15], [16]. Finally, this work is organized as follows: section 2 submits our proposed routing protocols with its work procedures in details, while section 3 demonstrates our experimental tests that will be done for testing our proposed routing protocols by given network example for each proposed routing protocol as a case study. Then, section 4 gives the main contributions and conclusions that we got from proposing these routing protocols and gives our thoughts for future works in this field of research in wireless networks.

\section{RESEARCH METHOD}

In this work, we will try to exploit the main advantages of famous wireless network routing protocols such as DSR, DSDV, OLSR and ZRP in order to create new routing protocols for achieving high performance gain than those traditional routing protocol [17]-[19]. The first proposed routing protocol in this research work comes from enhancing DSDV routing protocol by adding more than one performance metric to find the optimal route path among nodes at same network, in this proposal routing protocol enhanced destination sequenced distance vector (E-DSDV), we consider more than one performance metrics such sequence number, number of hops, and cost. Then, we give priority level for each performance metric, for example we can consider the sequence number has more priority than number of hops when we want to choose two existing paths since the path that has largest sequence number meaning this path is more recent updated path so we can avoid routing loops through finding the route to the destination node. The second proposed routing protocol comes from merging DSDV and ZRP routing protocols [20], [21], so we have destination-sequenced zone routing protocol (DS-ZRP) new routing protocol. At this protocol, the optimal route can be found by considering destination sequenced number from DSDV routing protocol and routing zone strategy from ZRP routing protocol, so we split the entire network to some zones and there are two types of nodes (interior zone nodes and exterior zone nodes), interior zone nodes use link state or distance vector routing protocols entire the zone considering sequence number as a performance metric, while the exterior zone nodes use DSR routing protocol to find the route outside the zone also considering sequence number to choose the more recent path to the destination node. The third proposed routing protocol comes from merging the advantages of OLSR and DSR routing protocols to have a new routing protocol named optimized link state dynamic source routing (OLS-DSR) routing protocol [22], [23]. At this new routing protocol, each node elects its multipoint relay (MPR) nodes from its neighbor nodes according to proposal zone radius after that the node floods only its link state information to its MPR nodes instead to all its neighbor nodes. Then, these MPR nodes use DSR routing protocol to flood the route cashing technology in this protocol, which gives instantly the path for intended route to the destination which it is the main advantage of DSR routing protocol.

\section{PROPOSED ROUTING PROTOCOLS}

In this section, we will describe in details the work procedure of each proposed routing protocol taking into account the main network performance metrics considered for each one of these protocol. Each proposed routing protocol has a work procedure related to its network behavior and we suppose that all hosts can communicate easily with each other in the given coverage area and it is assumed also all links between hosts are in full duplex communication mode. For our network model, we assume that hosts don't know any things about their location, direction, or nearby nodes. Since we considered a multi-hop network model, then, we suppose the link costs among hosts may determine using sequence number and number of hops respectively.

\subsection{First proposed enhanced routing protocol}

This work considers one of the famous routing protocols for mobile ad hoc networks-namely: DSDV which it is examined against two important metrics as sequence number and number of hops in order to deliver the sender's messages to the recipient with minimal time and the reliable path possible. This work 
comes with proposing a creative method of calculating optimal routing path by enhancing DSDV routing so we called it enhanced DSDV routing protocol (EDSDV), this approach procedure is based originally on Bellman-Ford algorithm and then sequence number to know whether this node has currently receive this message or not so as avoiding unproductive routing loops. In our enhanced routing protocol, nodes can work together to obtain an objective opinion about the credibility of another node. The work procedure of our proposed routing protocol EDSDV describes as follows as shown in Figure 1:

a) Step 1: select source node; in this step, we must choose one node as a source node to other destination nodes, so we can begin our search mission to find the optimal path to all other network nodes except selected source node.

b) Step 2: initial routing attribute; each node instantly has its own routing paths to other nodes, so we can screenshot this current initial route attribute for our selected source node to find the next optimal paths to same other nodes.

c) Step 3: attributes evaluation; in this work, we consider each node's routing table has two attributes (sequence no. and number of hops or cost). In this step, we will give the priority for node which has largest sequence number rather than number of hops which means this node has more recent update information (i.e. its routing table is updated recently), so new joining or leaving nodes in wireless network are considered instantly.

d) Step 4: update decision; in this step, we will update the routing table attributes according to sequence number firstly and then according to the number of hops as shown in the following cases:

- If sequence number of any node is greater than all other nodes, then the next path for our source node will be via this node regardless the number of hops for these nodes.

- If the sequence numbers of two nodes or more are equal, then we will consider the node that has less number of hops to be via node for next path of our source node.

- If the sequence numbers of two nodes or more are equal and the number of hops of same two nodes or more are also equal, then we will consider the current existed node as via node for the next path of our source node.

e) Step 5: new attributes updated; step 4 is repeated for all outgoing links from our source node and then new attributes are updated accordingly for source node.

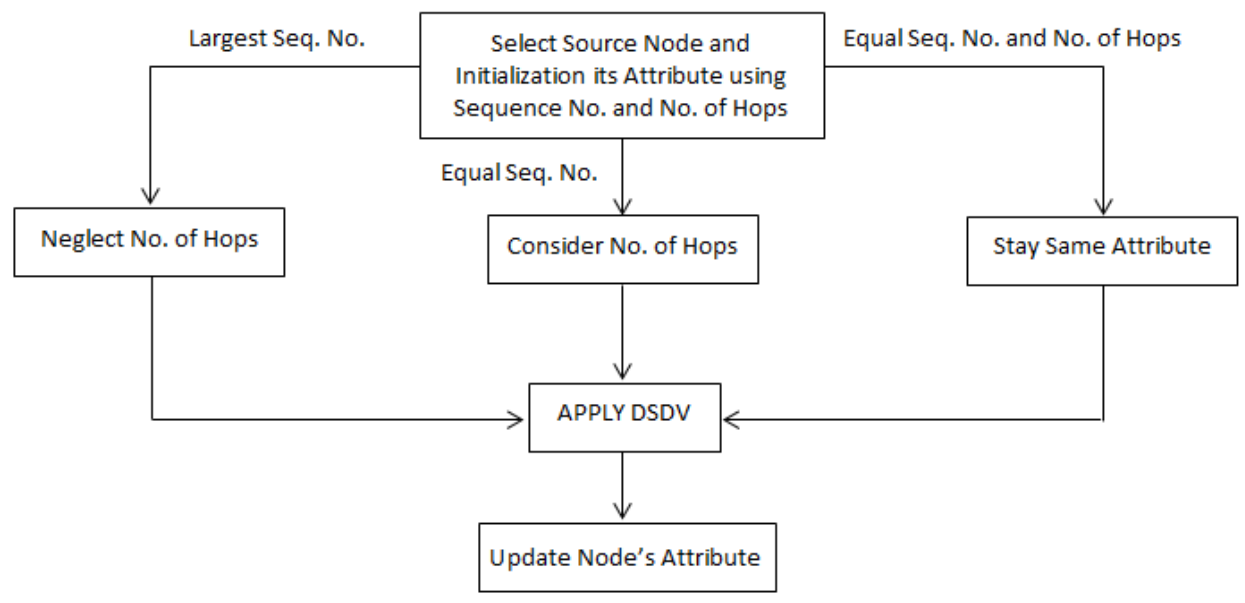

Figure 1. Effects of selecting different switching under dynamic condition

\subsection{Second proposed emerged and enhanced routing protocol}

In this proposed routing protocol, we merge the advantages of two known routing protocols DSDV and ZRP, where DSDV has two main advantages: 1) all nodes use sequence number to update their routing tables around all neighbour nodes occasionally, and then they increment and append their sequence numbers when sending their local routing tables which this sequence number will be attached to route entries created for this node; 2) this protocol has a low latency time since the routing table was proactively initiated and no need to new routing determinations. In contrast, ZRP has a main feature of combining two routing behaviors (proactive and reactive) in his work to find the optimal path from source to destination. This protocol determines paths to neighbors' nodes within minimum number of hops via either link state routing protocol or distance vector routing protocol for interior nodes within same zone, while it uses a route discovery 
protocol for determining routes to exterior (peripheral) nodes outside the source node's zone. Thus, ZRP has the following two main advantages; 1) diminishing the control burden for extended paths that will be important if using proactive routing protocols are used across the path, 2) eliminate routing delays within a region that might be happened by path detections for interactive routing protocols. So, our proposed routing protocol DS-ZRP merges all above advantages of these two protocols which its work procedure describes as follows as shown in Figure 2:

a) Step 1: network Initialization. Let $\mathrm{N}$ be the number of nodes at a multihop wireless network, and suppose the source node is $(\mathrm{S})$ and the destination node is $(\mathrm{D})$.

b) Step 2: intra-zone routing evaluation. Is destination node (D) within node (S) zone? If yes, we can apply Intra-zone routing using distance vector or link state routing protocol to reach destination node (D). Now, to know if the path to an intended destination node is more recent, destination sequence numbers are verified and considered (i.e. DSDV routing protocol). Any nearby node that has a route request within a smaller sequence number, it cannot forward this route request and so on.

c) Step 3: inter-zone routing evaluation: Is destination node (D) within node (S) zone? If no, we can apply Inter-zone routing using DSR protocol to reach destination node (D) as follows:

- Node (S) goes to publish a route query (RREQ) to all its exterior nodes outside the source node's zone using DSR protocol.

- To know if the path to an intended destination node is more recent, destination sequence numbers are verified and any nearby node that has a route request within a smaller sequence number, it cannot forward this route request.

- When the RREQ reaches the peripheral or exterior nodes, they will also apply the same previous procedure to check whether target host (D) located at same zone or not.

- Through all routing path, any host node detects the destination node (D) within its same region and then returns the route reply (RREP) by reversing the RREQ path.

d) Step 4: data transfer. After complete above steps, data is transferred along the new initiated optimal path for our selected source node.

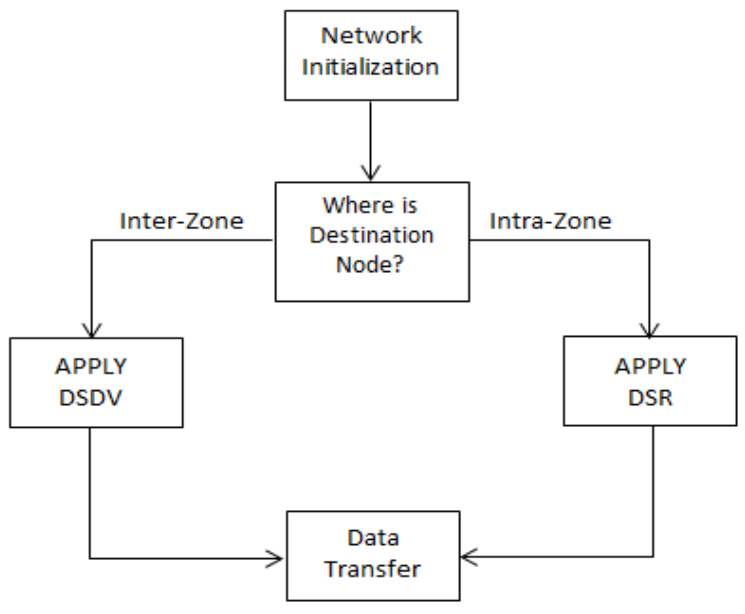

Figure 2. Proposed DS-ZRP routing protocol diagram

\subsection{Third proposed emerged and enhanced routing protocol}

In this proposal protocol, a routing process that can merge the advantages of using multipoint relays in optimized link state routing (OSLR) protocol with the advantages of route cashing in DSR protocol. The main advantage of using multipoint relays is that the overhead of flooding link state information is reduced by requiring fewer nodes (multipoint relay-MPR) to forward the information instead of broadcasting information to all neighbor nodes. While using of route cache can speed up route discovery and it can reduce propagation of route requests since a single route discovery may yield many routes to the destination, due to intermediate nodes replying from local caches. The work procedure of our proposed routing protocol OLSDSR can be described in the following steps as shown in Figure 3:

a) Step 1: network initialization. Let $\mathrm{N}$ be the number of nodes of multi-hop wireless network and let the source node is $(\mathrm{S})$ and the destination node is $(\mathrm{D})$. 
b) Step 2: initiating route discovery. Source node (S) floods RREQ to specify its multipoint relay (MPR) so that the RREQ forwards through it. Assuming the multipoint relay is with zone radius $r$-hops neighbor nodes.

c) Step 3: intra-zone routing evaluation. Is the destination node (D) within zone radius (r-hops)? If yes, apply (RREQ) directly to get more recent destination path by the use of sequence number (SN), if the sequence number at any node is less than that of any node, it cannot send RREP.

d) Step 4: inter-zone routing evaluation: If the target host (D) isn't around the zone's source node (S) (i.e. the distance is greater than r-hops), then route cashing is performed with radius (r-hops) in order to specify the multipoint relays.

e) Step 5: multipoint relays selection. Using route cashing strategy, the greater paths are reduced and then network overhead is damped since it stores only the most recent multipoint relays, note that we should notice that RREQ by source node (S) for destination node (D) is assigned a higher destination sequence number and then any intermediate node that receives a route request with a smaller sequence number, it cannot forward this route request and so on.

f) Step 6: initiating route reply. Now, the destination node (D) is known by the multipoint relays, then RREP is reversed according to the evaluation of the pair MPR/SN.

g) Step 7: data transfer. After the completion of RREP process, data transfer can be taken place now

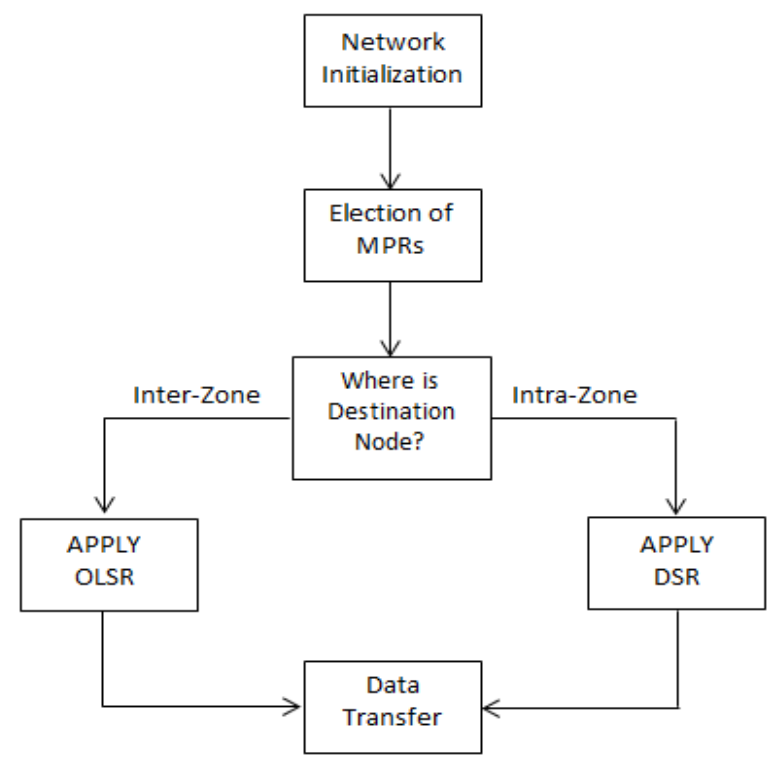

Figure 3. Proposed OLS-DSR routing protocol diagram

\section{RESULTS AND DISCUSSION}

Before presenting the experimental results, we first introduced the background details of network assumptions, network constraints and performance parameters. Below are the hypotheses constraints are considered to do our experimental results for the proposed routing protocols [24], [25]:

- All hosts contain perfect communication coverage opportunities assuming they are existed in a $2 \mathrm{D}$ space.

- All hosts are located in same coverage ranges assuming the communication links are in full-duplex mode.

- Our network model is assumed to be a multi-hop network.

- Each host doesn't have any information about its location, direction, or nearby nodes.

- Link costs of hosts determine using sequence number and number of hops consequently.

- The proposed emerged and enhanced routing protocols must work in a decentered way.

- Network under consideration is assumed to have a low message and computational complexity.

- For given network structure, the tested proposed routing protocol must cover the area of interest with fewer hosts as possible.

So, the evaluations of our proposed emerged and enhanced routing protocols are considering the following network performance metrics: 
- A number of nodes: this metric specifies whole wireless network size in average because there are some nodes joining or leaving instantly. To simplify the mathematical computation and analysis; we do our experimental test with less than 50 nodes.

- A number of hops: this metric defines the cost or distance between one node and other and also to simplify the calculations, we assumed small number of hops among different nodes in each considered network topology.

- Sequence number: this number is also called destination sequence number which it is applied to prohibit paths from going into infinity associated with classic distance vector routing protocols. So, the path that has largest sequence number meaning this path is more recent updated path.

- Routing zone: some routing protocols used routing zone in order to minimize the flooding route requests. Accordingly, nodes are divided into two types: interior and exterior (peripheral) nodes. The interior nodes are the nodes whose are located inside the routing zone where routing zone radius equals $d$ hops and these nodes used link state or distance vector routing protocols. While the exterior nodes are those nodes that are located outside routing zone radius and they are used DSR routing protocol.

Now, we consider the network topology shown in Figure 4 to demonstrate and do first experimental test for E-DSDV. In this experimental test, we will try to construct a new routing table for node $\left(\mathrm{n}_{4}\right)$ using our proposed E-DSDV routing table. The new routing table for node $\left(\mathrm{n}_{4}\right)$ is constructed by applying E-DSDV shown in Table 1.

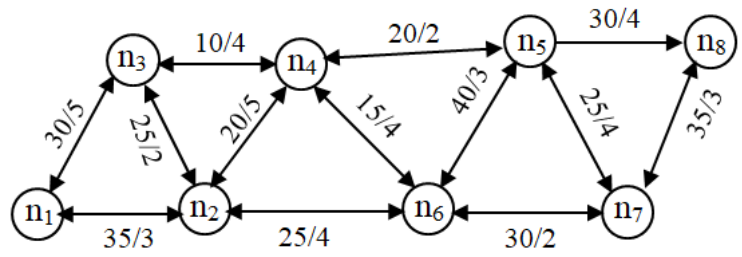

Figure 4. Network topology for applying E-DSDV routing protocol

Table 1. Routing table determination for $\mathrm{n}_{4}$ using E-DSDV routing protocol

\begin{tabular}{cccccccccc}
\hline \multicolumn{2}{c}{ Current Distance Vector } & \multicolumn{4}{c}{ Directed Neighbor Nodes } & \multicolumn{2}{c}{ New Distance Vector } \\
\hline Dest. & Cost & $\mathrm{SN}$ & $\mathrm{By}_{2}$ & $\mathrm{By}_{3}$ & $\mathrm{By}_{5}$ & $\mathrm{By}_{6}$ & Cost & SN & Via \\
\hline $\mathrm{n}_{1}$ & - & - & $55 / 5$ & $40 / 4$ & - & - & 55 & 5 & $\mathrm{n}_{2}$ \\
$\mathrm{n}_{2}$ & 20 & 5 & $20 / 5$ & $35 / 4$ & - & - & 20 & 5 & $\mathrm{n}_{2}$ \\
$\mathrm{n}_{3}$ & 10 & 4 & $45 / 5$ & $10 / 4$ & - & - & 45 & 5 & $\mathrm{n}_{2}$ \\
$\mathrm{n}_{5}$ & 20 & 2 & - & - & $20 / 2$ & $55 / 4$ & 55 & 4 & $\mathrm{n}_{6}$ \\
$\mathrm{n}_{6}$ & 15 & 4 & $45 / 5$ & - & $60 / 2$ & $15 / 4$ & 45 & 5 & $\mathrm{n}_{2}$ \\
$\mathrm{n}_{7}$ & - & - & - & - & $45 / 2$ & $45 / 4$ & 45 & 4 & $\mathrm{n}_{6}$ \\
$\mathrm{n}_{8}$ & - & - & - & - & $50 / 2$ & - & 50 & 2 & $\mathrm{n}_{5}$ \\
\hline
\end{tabular}

For second experimental test, we supposed to have a network topology as shown in Figure 5 in order to test the work of our second proposed routing destination-sequenced zone routing protocol (DS-ZRP), and as a case study, the destination (D) is not within node (s) zone, so we will apply step 3 of above work procedure to find the route from source node (s) to destination node (D) assuming the network performance metric is a sequence number with zone radius is 2 . So, we will split the entire network into some zones with zone radius (2) and they will be two types of nodes: interior zone nodes and exterior (peripheral) zone nodes. The interior zone nodes use link state or distance vector routing protocols inside the zone taking into account the sequence number as a performance metric, while the peripheral zone nodes use DSR routing protocol to find the route outside the zone also taking into account the sequence number to choose the more recent path to the destination node. Then, the work procedure of applying DS-ZRP routing protocol can be described as follows as shown in Figure 6:

- Node (S) sends RREQ to all its peripheral nodes ((C, D, E, F, H)) using DSR routing protocol.

- Using DSDV routing protocol, only node (C) forwarding RREQ since it has a higher destination sequence number.

- Then, node (C) sends RREQ to all its peripheral nodes ((J, K, I)) using DSR routing protocol.

- Using DSDV routing protocol, only node (K) forwarding RREQ since it has a higher destination sequence number.

Then, node (K) find out the destination host (D) is within same its zone and then return RREP by reversing the RREQ path such as [K-C-S]. 


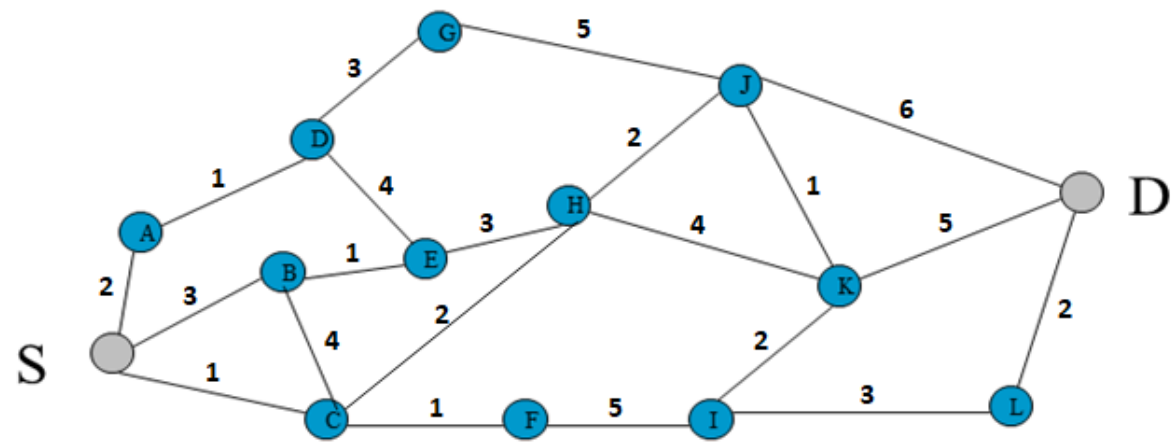

Figure 5. Network topology for applying DS-ZRP routing protocol

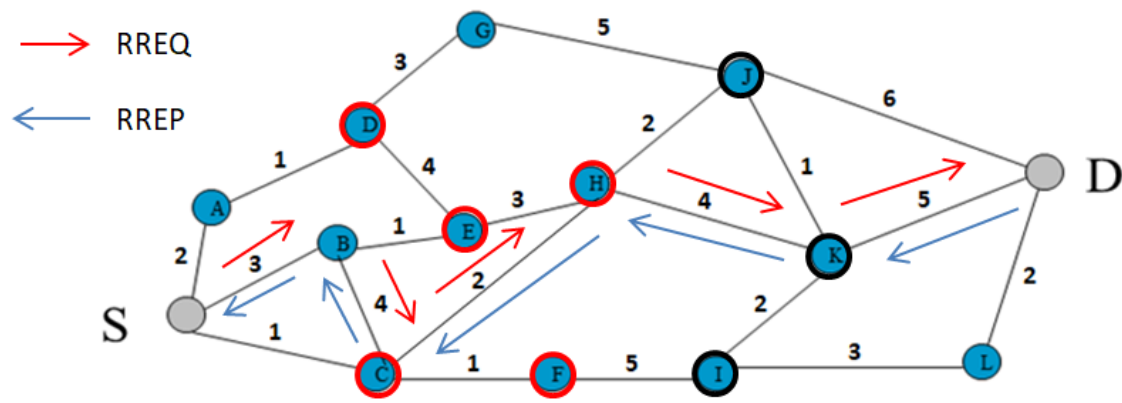

Figure 6. Optimal route from source node (S) to destination node (D) using DS-ZRP routing protocol

In third experimental test, we will check our last proposed routing protocol an OLS-DSR. We consider the network topology shown in Figure 7 to do our test and we will explain the using of this proposed routing protocol by the following steps below and then solve a demonstration example using the above network for sending packets from node (S) to node (D), considering the network performance metric is the sequence number with zone radius is 2 .

- $\quad$ Node (S) sends RREQ to all its multipoint relays so it will send RREQ to nodes (A, F) as shown in Figure 7.

- Since the RREQ passes through node $(\mathrm{C})$, so it is also forward it to node $(\mathrm{H})$.

- The route reply will be in reverse direction and containing pairs of multihops relays and higher destination sequence number. For example, let us take the path for RREQ as (C, F, I, L)-most recent updated sequence numbers and the route reply as $[\mathrm{C} / 5, \mathrm{~F} / 5, \mathrm{I} / 7, \mathrm{~L} / 11]$ which it represents the header of path contains pair of [Node/SN].

- Now, data can be sent over this path $[\mathrm{C} / 5, \mathrm{~F} / 5, \mathrm{I} / 7, \mathrm{~L} / 11]$ as described in Figure 7.

- Assume $\mathrm{SN}_{\mathrm{n}}$ is the destination sequence number at node (n). We will assume sequence numbers in order to clarify the concept of proposed routing protocol.

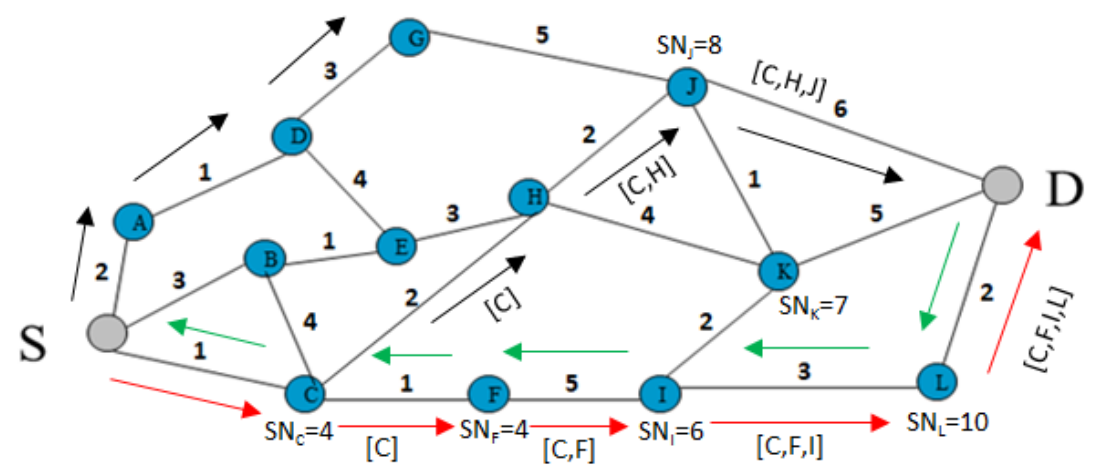

Figure 7. Optimal route from source node (S) to destination node (D) using OSL-DSR routing protocol $[\mathrm{C} / 5, \mathrm{~F} / 5, \mathrm{I} / 7, \mathrm{~L} / 11]$ 
In Table 2 shows a comparison among these proposed routing protocols versus traditional routing protocols considering main network performance metrics such as periodical broadcast, network control overhead, bandwidth overhead, energy consumed and latency. This table shows that our proposed routing protocols outperform than traditional routing protocol working separately.

Table 2. A comparison among proposed emerged enhanced routing protocols vs. tradition routing protocols

\begin{tabular}{cccccc}
\hline Routing Protocol & Periodical Broadcast & Network Control Overhead & Bandwidth Overhead & Energy Consumed & Latency \\
\hline DSDV & HIGH & HIGH & HIGH & HIGH & MEDIUM \\
DSR & HIGH & MEDIUM & MEDIUM & MEDIUM & HIGH \\
OLSR & HIGH & HIGH & HIGH & HIGH & HIGH \\
ZRP & MEDIUM & MEDIUM & MEDIUM & HIGH & MEDIUM \\
E-DSDV & LOW & MEDIUM & HIGH & MEDIUM & LOW \\
DS-ZRP & LOW & MEDIUM & MEDIUM & LOW & LOW \\
OLS-DSR & LOW & LOW & LOW & LOW & LOW \\
\hline
\end{tabular}

\section{CONCLUSION}

In this work, we will try to exploit the main benefits from known wireless network routing protocols such as DSR, DSDV, OLSR and ZRP in order to propose new emerged enhanced routing protocols for achieving high performance gain than those traditional wireless network routing protocol. The first proposed routing protocol in this research work comes from enhancing DSDV routing protocol by adding more than one performance metric to find the optimal route path among nodes at same network, in this proposal routing protocol E-DSDV, we consider more than one performance metrics such sequence number, number of hops, and cost. The second proposed routing protocol comes from merging DSDV and ZRP routing protocols so we have DS-ZRP new routing protocol. At this protocol, the optimal route can be found by considering destination sequenced number from DSDV routing protocol and routing zone strategy from ZRP routing protocol. The third proposed routing protocol comes from merging the advantages of OLSR and DSR routing protocols to have a new routing protocol named OLS-DSR routing protocol. At this new routing protocol, each node elects its MPR nodes from its neighbor nodes according to proposal zone radius after that the node floods only its link state information to its MPR nodes instead to all its neighbor nodes. Then, these MPR nodes use DSR routing protocol to flood the route cashing technology in this protocol, which gives instantly the path for intended route to the destination which it is the main advantage of DSR routing protocol.

\section{REFERENCES}

[1] S. K. Das, S. Samanta, N. Dey, and R. Kumar, "Design frameworks for wireless networks," In Networks and Systems, Springer, Singapore, 2020, doi: 10.1007/978-981-13-9574-1.

[2] M. Z. Chowdhury, M. Shahjalal, S. Ahmed, and Y.M. Jang, "6G wireless communication systems: Applications, requirements, technologies, challenges, and research directions," IEEE Open Journal of the Communications Society, vol. 1, pp. 957-975, 2020, doi: 10.1109/ojcoms.2020.3010270.

[3] N. H. Alkhazaali, R. A. Aljiznawi, S. Q. Jabbar, and D. J. Kadhim, "Mobile Communication through 5G Technology (Challenges and Requirements)," International Journal of Communications, Network and System Sciences, vol. 10, no. 5, pp. 202-207, 2017, doi: 10.4236/ijcns.2017.105b020.

[4] H. N. Dai, R. C. W. Wong, H. Wang, Z. Zheng, and A. V. Vasilakos, "Big data analytics for large-scale wireless networks," ACM Computing Surveys (CSUR), vol. 52, no. 5, pp. 1-36, 2019, doi: 10.1145/3337065.

[5] D. J. Kadhim and O. A. Hamad, "Improving IoT Applications Using a Proposed Routing Protocol," Journal of Engineering, vol. 20, no. 11, pp. 50-62, 2014.

[6] W. A. Mahmoud and D. J. Kadhim, "A Proposal Algorithm to Solve Delay Constraint Least Cost Optimization Problem,” Journal of Engineering, vol. 19, no. 1, pp. 155-160, 2013.

[7] J. Yi, A. Adnane, S. David, and B. Parrein, "Multipath optimized link state routing for mobile ad hoc networks. Ad hoc networks," Ad Hoc Networks, vol. 9, no. 1, pp. 28-47, Jan. 2011, doi: 10.1016/j.adhoc.2010.04.007.

[8] S. Kalaivanan, "Quality of service (QoS) and priority aware models for energy efficient and demand routing procedure in mobile ad hoc networks," Journal of Ambient Intelligence and Humanized Computing, vol. 12, no. 3, pp. 1-8, Mar. 2021, doi: 10.1007/s12652-020-01769-7.

[9] C. Anastasiades, J. Weber, and T. Braun, "Dynamic unicast: Information-centric multi-hop routing for mobile adhoc networks," Computer Networks, vol. 107, pp. 208-219, Oct. 2016, doi: 10.1016/j.comnet.2016.03.009.

[10] S. A. Lafta, A. H. Ali, M. M. Kareem, Y. A. Hussein, and A. H. Ali, "Performance simulation of broadband multimedia wireless networks simulation based on OPNET," Indonesian Journal of Electrical Engineering and Computer Science (IJEECS), vol. 17, no. 1, pp. 1-9, Jan. 2020, doi: 10.11591/ijeecs.v17.i1.pp1-9.

[11] O. A. Mahdi, Y. R. B. Al-Mayouf, A. B. Ghazi, A. W. A. Wahab, and M. Y. I. B. Idris, "An Energy-Aware and Load-balancing Routing Scheme for Wireless Sensor Networks," Indonesian Journal of Electrical Engineering and Computer Science (IJEECS), vol. 12, no. 3, pp. 1312-1319, Dec. 2018, doi: 10.11591/ijeecs.v12.i3.pp1312-1319. 
[12] M. A. Jubair et al., "Competitive analysis of single and multi-path routing protocols in mobile Ad-Hoc network," Indonesian Journal of Electrical Engineering and Computer Science (IJEECS), vol. 14, no. 2, pp. 203-300, Jul. 2020, doi: 10.11591/ijeecs.v19.i1.pp293-300.

[13] M. Abbas, H. M. T. Alhilfi, and T. Sutikno, "Performance evaluation of two models in the reactive routing protocol in manets," Indonesian Journal of Electrical Engineering and Computer Science (IJEECS), vol. 21, no. 1, pp.391397, Jan. 2021, doi: 10.11591/ijeecs.v21.i1.pp391-397.

[14] D. J. Kadhim, S. S. Abed and S. Q. Jabbar, "Performance Evaluation of AODV Routing Protocol in MANET using OPNET Simulator," Anbar Journal of Engineering Sciences, vol. 5, no. 2, pp. 241-257, 2012.

[15] W. M. Lafta, S. Q. Jabbar, D. J. Kadhim, and G. Ma, "Heterogeneous Network Performance Improvement Using Proposed OLRED and OLWRED Strategies," International Journal of Future Computer and Communication, vol. 5, no. 5, pp.199-204, 2016, doi: 10.18178/ijfcc.2016.5.5.471.

[16] L. M. Bendale, R. L. Jain, and G. D. Patil, "Study of various routing protocols in mobile ad-hoc networks," International Journal of Scientific Research in Network Security and Communication, vol. 6, no. 1, pp. 5-15, 2018.

[17] D. J. Kadhim, "A Proposed Solution for Route Reply Storm Problem to Improve DSR Protocol Performance in Wireless Sensor Networks," IOP Conference Series: Materials Science and Engineering, vol. 1076, no. 1, 2021, doi: 10.1088/1757-899x/1076/1/012058.

[18] A. Singh, G. Singh and M. Singh, "Comparative study of OLSR, DSDV, AODV, DSR and ZRP routing protocols under blackhole attack in mobile ad hoc network," In Intelligent Communication, control and devices, 2018, pp. 443-453, doi: 10.1007/978-981-10-5903-2_45.

[19] F. T. AL-Dhief, N. Sabri, M. S. Salim, S. Fouad, and S. A. Aljunid, "MANET routing protocols evaluation: AODV, DSR and DSDV perspective," In MATEC web of conferences, vol. 150, 2018, doi: $10.1051 /$ matecconf $/ 201815006024$.

[20] Y. Fengjie, Y. Hui, and Z. Ying, "Research on DSDV routing protocol based on wireless Mesh network," In 2018 Chinese Control And Decision Conference (CCDC), Jun. 2018, pp. 4292-4297, doi: 10.1109/ccdc.2018.8407870.

[21] R. R. Chandan, B. S. Kushwaha, and P. K. Mishra, "Performance Evaluation of AODV, DSDV, OLSR Routing Protocols using NS-3 Simulator," International Journal of Computer Network \& Information Security, vol. 10, no. 7, pp. 59-65, Jul. 2018, doi: 10.5815/ijcnis.2018.07.07.

[22] P. Lavanya, V. S. K. Reddy and A. M. Prasad, "Performance Comparison of DSDV, OLSR, AODV and DSR for Mobile Ad hoc Networks," International Journal of Emerging Technology and Advanced Engineering, vol. 8, no. 1, pp. 209-218, 2018.

[23] D. J. Kadhim and N. A. Rahman, "Performance analysis of xPON network for different queuing models," International Journal of Electrical, Electronics and Telecommunication Engineering, vol. 44, no.1, pp. 1131-1317, 2013.

[24] S. Q. Jabbar, D. J. Kadhim and Y. Li, "Developing a video buffer framework for video streaming in cellular networks," Wireless Communications and Mobile Computing, 2018, doi: doi.org/10.1155/2018/6584845.

[25] M. Alhihi, "Practical routing protocol models to improve network performance and adequacy," Journal of Computer and Communications, vol. 5, no. 6, pp.114-124, 2017, doi: 10.4236/jcc.2017.56007. 